\title{
In Silico Discovery of High Deliverable Capacity Metal-Organic Frameworks
}

\author{
Yi Bao, ${ }^{\dagger}$ Richard L. Martin, ${ }^{\ddagger}$ Cory Simon, $₫$ Maciej Haranczyk, ${ }^{\ddagger}$ Berend Smit, $₫$ \\ and Michael W. Deem ${ }^{*}, \dagger$ \\ Department of Physics 83 Astronomy, Rice University, Houston, TX, 77005, Computational \\ Research Division, Lawrence Berkeley National Laboratory, Berkeley, CA, 94720, \\ Department of Chemical \& Biomolecular Engineering, University of California, Berkeley, \\ CA, 94720, and Department of Bioengineering, Rice University, Houston, TX, 77005 \\ E-mail: mwdeem@rice.edu
}

\footnotetext{
${ }^{*}$ To whom correspondence should be addressed

${ }^{\dagger}$ Rice University

${ }^{\ddagger}$ Lawrence Berkeley National Laboratory

ฯ University of California, Berkeley

${ }^{\S}$ Rice University
} 


\begin{abstract}
Metal organic frameworks (MOFs) are actively being explored as potential adsorbed natural gas storage materials for small vehicles. Experimental exploration of potential materials is limited by the throughput of synthetic chemistry. We here describe a computational methodology to complement and guide these experimental efforts. The method uses known chemical transformations in silico to identify MOFs with high methane deliverable capacity. The procedure explicitly considers synthesizability with geometric requirements on organic linkers. We efficiently search the composition and conformation space of organic linkers for nine MOF networks, finding 48 materials with higher predicted deliverable capacity (at 65 bar storage, 5.8 bar depletion, and $298 \mathrm{~K})$ than MOF-5 in four of the nine networks. The best material has a predicted deliverable capacity $8 \%$ higher than that of MOF-5.
\end{abstract}

\title{
Keywords
}

MOF, methane storage, deliverable capacity 


\section{Introduction}

MOFs are coordination networks consisting of metal ions or metal clusters, termed as secondary building units (SBUs), and organic binding ligands, termed as linkers. MOFs were first described in the literature in $1995 .{ }^{1}$ After two decades of contributions, the number of synthesized MOFs amount to several thousand. ${ }^{2}$ Due to their high porosities, high surface area, and tunable chemistry, MOFs are regarded as a promising class of nano-porous ma-

terials. ${ }^{3}$ Potential applications of MOFs include drug delivery, ${ }^{4}$ sensing, ${ }^{5,6}$ purification, ${ }^{7-9}$ catalysis, ${ }^{10}$ and gas storage. ${ }^{11,12}$ In the gas storage application, in particular, MOFs appeal as a competitive alternative to other materials, such as zeolites, because of their potentially higher performance and adjustability. ${ }^{6,7,13}$ Computation predictions have extended the number of potential MOFs to $>100,000 \cdot{ }^{14}$

From a practical point of view, one can only synthesize and test a small fraction of all possible MOF materials, and computation predictions are useful for suggesting promising sets of MOFs to synthesize. Existing prediction methods focus on simulation of the selfassembly process, placing known SBUs into candidate periodic networks. ${ }^{14-16}$ A limitation of these methods is that only pre-existing linkers are considered, with little or no exploration of the space of possible organic linkers.

We here develop a de novo evolutionary algorithm to explore the composition and configuration space of linker molecules to optimize methane deliverable capacity in predicted MOFs. Since the linker, SBU, and topology can all vary, the chemical search space is nearly infinite. This poses a fundamental problem for the current methods of library generation, which are all based on brute force enumeration, as the number of compounds grows exponentially with length and branching structure of the linkers. The vast majority of these potential have poor methane delivery performance. Thus, it is desirable to efficiently sample the part of the MOF composition space with favorable materials properties. We tackle this issue by using an evolutionary algorithm to rapidly explore MOF linker composition space, among MOFs with high predicted methane deliverable capacity. Our approach keeps track 
of the synthetic route of each proposed linker, with chemical reactants and corresponding reactions, so that the linkers optimized for methane storage are also experimentally synthesizable. The method described here is inspired both by the "biased Monte Carlo" methods that revolutionized computer simulation ${ }^{17}$ and by the drug design field, in which over 55 lead compounds in various stages of clinical trials have been identified by in silico prediction approaches. ${ }^{18}$

In this work, we predict MOFs with high methane deliverable capacity in nine different networks, each formed with a specified SBU. For each network and chosen SBU, we evolve a population of linker molecules and build MOFs to optimize methane deliverable capacity. As will be described more fully below, the set of linker molecules is initialized from a set of commercially available compounds. The linker molecules are evolved in silico by a set of chemical reactions, so that only chemically-synthesizable linkers are considered as the deliverable capacity is optimized. These in silico reactions are known to succeed with high probability and high yield. ${ }^{19}$

Among the materials we predict are ones with a higher deliverable capacity than MOF5 (i.e., IRMOF-1), a well known MOF with a high methane deliverable capacity. The experimentally measured capacity of MOF-5 is $177 \mathrm{v}(\mathrm{STP}) / \mathrm{v}$ at 65 bar adsorption pressure,

5.8 bar delivery pressure, and $298 \mathrm{~K}^{20-22}$ In all, we find 48 MOFs with a greater deliverable capacity than MOF-5: 14 in acs, 24 in $\mathbf{c d s}, 2$ in $\mathbf{n b o}$, and 8 in $\mathbf{p c u}$, with the best deliverable capacity found in pcu and $8 \%$ greater than MOF-5.

\section{Methods}

In general, we select nine well known networks, acs, cds, dia, hxg, lvt, nbo, pcu, rhr, and sod, to search for MOFs with a high methane deliverable capacity. We assume that each MOF consists of one type of SBU and one type of linker molecule. To optimize deliverable capacity, we explore the compositional space of linker molecules. We require that each linker 
has two carboxylic groups to connect with exactly two SBUs. The flowchart of the evolution algorithm is illustrated in Fig. 1.

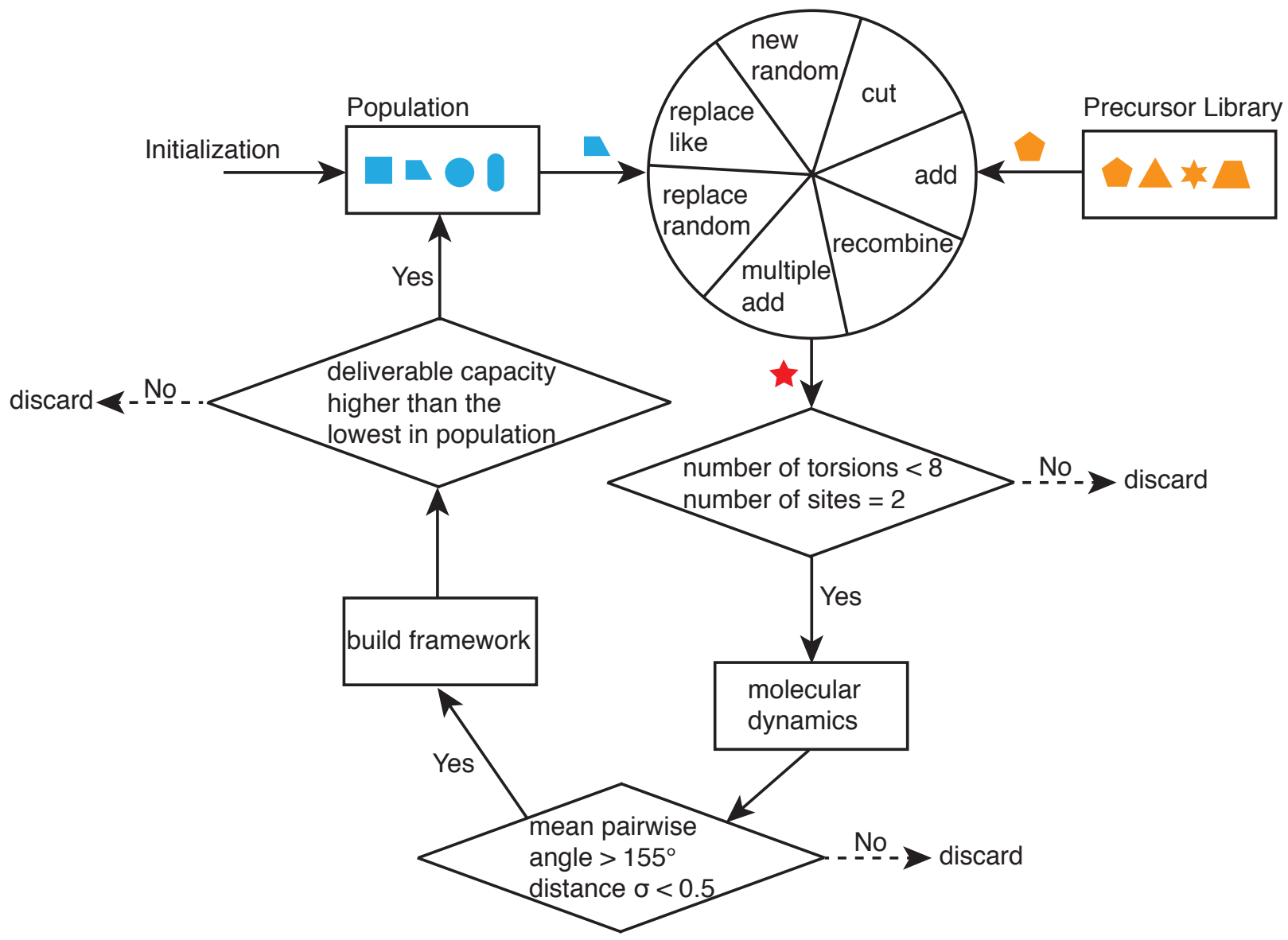

Figure 1: Illustration of the algorithm. The precursor library is used for initialization, and add and multiple add operation. One randomly chosen linker from the population undergoes one of seven evolution operations. The produced linker is evaluated by two filters first, i.e. number of torsions $<8$ and number of sites $=2$. If the linker passes these filters, it undergoes molecular dynamics to produce a set of conformations. These linker conformations are evaluated by the two additional filters of mean pairwise angle $\geq 155^{\circ}$ and standard deviation of pairwise distance $<0.5 \AA$. If the linker passes all filters, it is used to build a MOF of the chosen network. Finally, if the constructed MOF has a greater deliverable capacity than the lowest one in the current population, the linker is inserted into the population in rank order.

We use an evolutionary algorithm to evolve the MOF structures to optimize the methane deliverable capacity. We use a population of 100 linker molecules, which is initialized by compounds from a precursor library of commercially available molecules. The precursor library contains next-day-available compounds from Sigma-Aldrich. It also contains compounds 
that are used in medicinal chemistry or as organic building blocks from Sigma-Aldrich. All the compounds amount to 56,931 molecules in total. Up to five chemical addition reactions may be applied to the linkers before they enter the initial population, because precursors directly drawn from the library may often lead to low deliverable capacity in the initial population. It is important to note that the population size remains 100 at all times after the initialization.

In each generation, one molecule undergoes one of seven evolution operations randomly producing a secondary molecule. One molecule is first randomly picked in the population. One of seven evolution operations is then uniformly and randomly chosen, and applied to the linker. If it is chemically impossible for the chosen operation to be applied to the linker, another operation will be chosen, until one is eventually performed. The evolution operations are as follows:

1. Add. One chemically applicable reaction is randomly chosen from a list of 84 entries. ${ }^{19}$ These reactions are adjusted from an original set of 70 reactions. ${ }^{23}$ If it is a zero-order reaction, no more reactants are needed; otherwise, if it is a first-order reaction, one more reactant is chosen randomly from the precursor library to join the reaction. Regardless of whether zero or first order, the reaction results in an addition to the chosen linker, and one reaction step is added to the end of synthetic route of the linker.

2. Cut. The last reaction step is removed from the synthetic route of the linker. In other words, the synthetic route of the linker is reversed by one step back toward its parent molecule.

3. Replace random. One reactant in the synthetic route is replaced by another from the precursor library that has suitable functionality for the reaction of that particular step.

4. Replace like. One reactant in the synthetic route is replaced by another that has the required functionality from the precursor library. In addition, the reactant and its replacement must be chemically similar within a tolerance. Chemical similarity 
is computed from a chemical fingerprint, discussed in detail below. The similarity threshold is set to 0.75 .

5. New random. The linker is replaced by a new, randomly chosen compound from the precursor library. Up to five chemical addition reactions may be applied to the newly chosen linker before it enters the population.

6. Recombine. Another linker in the current population that shares at least one combinative node in the synthetic route with the linker to be evolved is randomly identified. The nodes denote where certain reactions with correspondent functionality groups occur. A child linker is created by choosing the initial part of the synthetic route from the first linker and the final part of the synthetic route from the second linker. In the case of more than one common combinative node in the synthetic route, one node is randomly chosen.

7. Multiple add. One chemically applicable reaction is randomly chosen. When multiple reaction sites are possible on one molecule, the reaction takes place on all these sites within one operation. One reaction step is added to the end of the synthetic route of the linker.

It is possible using these operations to produce a molecule that has previously been created during the run. In this case, a new molecule from the population is picked, and the evolution step restarts. A hash log is used to record all molecules produced.

After a linker has gone through an evolution operation, selection determines whether it is reinserted to the population or discarded. The selection is based on a five-component vector with four binary filters, and one real number. These five components are evaluated sequentially, as follows:

1. Number of torsions: binary filter. The number of torsions is required to be no more than eight. Too many torsions cause excessive flexibility. 
2. Number of carboxylic sites: binary filter. The number of carboxylic sites of the molecule is required to be exactly two. The metal SBUs examined in this work may give rise to alternative networks when combined with organic linkers with more than two connection sites; here we restrict our search to the aforementioned networks and hence to linear two-connected linkers.

3. Mean pairwise angle: binary filter. After the first two filters are passed, a 30 ps molecular dynamics simulation at $298 \mathrm{~K}$ on the molecule is performed to produce a set of 300 conformations. The mean pairwise angle over all conformations produced from molecule dynamics is required to be larger than a cutoff. The angle is defined as that between two vectors, one orienting from the carbon atom and passing through the midpoint of two oxygen atoms for the first carboxylic group metal attachment site and the other for the second carboxylic attachment site. It is anticipated that successful linkers will be linear, with anti-parallel attachment sites, and so desired angles are set as $\geq 155^{\circ}$.

4. Standard deviation of pairwise distance: binary filter. To avoid an entropic penalty against incorporation of the linkers into the MOF structure, rigid linkers are preferred. Such rigid linkers are more likely to successfully self-assemble the MOF structure. To quantify rigidity, we measure the standard deviation of pairwise distance between linker sites. By calculating several typical linear organic linkers of SURMOF-2, ${ }^{24}$ we find that the largest standard deviation of pairwise distance is $0.24 \AA$. In this study we used a conservative standard deviation of pairwise distance of $0.5 \AA$ as our cutoff. The distance is defined according to the coordinates of the carbon atoms in each of the carboxylic sites. The standard deviation is evaluated over all conformations produced in the molecular dynamics run.

5. Methane deliverable capacity: real. If the linker passes the filters above, a MOF is built using the linker. ${ }^{25,26}$ The fifth component of the vector is a measure of methane 
deliverable capacity. We use grand canonical Monte Carlo to simulate equilibrium methane uptake process in given frameworks at temperature of $298 \mathrm{~K}$, and to calculate the deliverable capacity. In each simulation, we construct a minimum supercell of the framework in three dimensions at an interaction radius cutoff of $12.5 \AA$. We use the universal force field to calculate interactions, including a tail correction. ${ }^{27}$ We calculate adsorption at the loading pressure of 65 bar and at the depletion pressure of 5.8 bar, both at $298 \mathrm{~K}$. The second result is subtracted from the first to give the deliverable capacity, i.e. the net excess loading between the two pressures. The reported values are multiplied by $R T_{0} / P_{0}$ where $T_{0}=273.15 \mathrm{~K}$ and $P_{0}=1$ bar to give units of $\mathrm{v}(\mathrm{STP}) / \mathrm{v}$. To maximize efficiency, we perform one methane uptake simulation with 300 Monte Carlo steps in total for every 50 linker conformations. Greater numbers of Monte Carlo steps did not significantly affect the average predicted deliverable capacity. The final deliverable capacity of each linker molecule is averaged over the conformations sampled in the molecular dynamics.

The filters are evaluated sequentially, as it is not necessary to evaluate further filters on a given proposed linker if one of the filters is not satisfied. The selection step operates hierarchically, comparing the first binary result, then the second, third, and fourth, and finally computing the deliverable capacity.

After the final child molecule has satisfied all filters, it will be inserted into the population in the rank order if its deliverable capacity is higher than the current worst molecule. Since the population size remains constant, this results in the worst molecule being discarded. Otherwise, the child molecule itself is discarded.

For each proposed linker, before molecular dynamics is carried out, an initial configuration is determined. Linkers are first minimized in terms of their configurational energy in isolation, outside the MOF, to find a local energy minimum. This sets the bond lengths and bond angles to equilibrium values. Then amide bonds are set into the trans isomer when applicable, i.e. $\mathrm{N}-\mathrm{H}$ being in a trans conformation with respect to $\mathrm{C}=\mathrm{O}$. This is done because molecules of 
the form $\mathrm{NHRC}(=\mathrm{O}) \mathrm{R}^{\prime}$ will be planar in general, and the trans conformation is more stable. Therefore, the amide torsions are restricted. Further exploration identifies an approximately global energy minimum state by using the ant algorithm. ${ }^{28}$

Molecular dynamics is carried out on each molecule to explore the flexibility and space of conformations to gauge their suitability as MOF linkers. The molecular modeling software TINKER $6.3,{ }^{29}$ is used to perform the molecular dynamics, if this molecule satisfies the first two filters. The dynamics is computed for $30 \mathrm{ps}$ at a temperature of $298 \mathrm{~K}$. The Merck Molecular Force Field commonly used in calculations on compounds in organic chemistry is used here. Conformations are sampled every 0.1 ps, giving a total of 300 conformations for each molecule.

A framework of the specified network is constructed for each conformation using the open source porous materials analysis suite Zeo $++{ }^{25}$ and model construction algorithm. ${ }^{26}$

We use a modified Langmuir model to characterize the MOFs ${ }^{30}$

$$
A=A_{\infty} \frac{P}{P+P_{1 / 2}}\left[1-\theta \frac{P P_{1 / 2}}{\left(P+P_{1 / 2}\right)^{2}}\right]
$$

where $P$ is pressure, $A_{\infty}$ is the saturation adsorption capacity of a MOF when the pressure goes to infinity, $\theta<0$ is a measure of methane-methane attractive interactions, and $P_{1 / 2}$ is the pressure at which adsorption capacity reaches one half of the maximum without the correction term, i.e. when $\theta=0$. To calculate the Languir adsorption isotherm parameter values, adsorption at 21 evenly spaced points between 0 and 100 bar was calculated. The $A_{\infty}$ and $P_{1 / 2}$ parameters were determined by fitting the Languir adsorption isotherm to these 21 data points by non-linear least-squares Marquardt-Levenberg algorithm.

We use a chemical fingerprint, a type of Tanimoto similarity coefficient, to define similarity between two molecules. Each molecule can be uniquely encoded into a bit string in terms of chemical structure. ${ }^{31}$ One string consists of 1092 bits, each denoting either absence (0) or presence (1) of a molecular motif. A motif is a selected single, double, triple or 
quadruple of types of atoms that are bonded, with types determined by the Merck Molecular Force Field. By calculating fingerprint similarities between each pair of two bit strings over all molecules, structural similarities can be quantitatively represented. Mathematically, fingerprint similarity $t$ for two bit strings $r$ and $s$ is defined as

$$
t(r, s)=\frac{\sum_{i} r_{i} \wedge s_{i}}{\sum_{i} r_{i} \vee s_{i}}
$$

where $r_{i}$ and $s_{i}$ are the $i$ th bit of $r$ and $s$, and $\wedge$ and $\vee$ are bitwise and and $o r$.

\section{Results}

The top deliverable capacity of each net is shown in Table 1, among which the pcu net gives highest deliverable capacity of $191.1 \mathrm{v}(\mathrm{STP}) / \mathrm{v}$. This value is $8 \%$ greater than our calculated value for MOF-5. As far as we know, predicted deliverable capacities significantly greater than MOF-5 under DOE target conditions have not been achieved before using any computational design approaches.

Table 1: Top deliverable capacity for each net. Candidates are defined as all molecules that have passed all filters and have a positive deliverable capacity during the whole evolution. With our force field, MOF-5 has a deliverable capacity of $177.6 \mathrm{v}(\mathrm{STP}) / \mathrm{v}$. The best predicted MOF has a deliverable capacity of $191.1 \mathrm{v}(\mathrm{STP}) / \mathrm{v}$.

\begin{tabular}{|c|c|c|}
\hline Net & $\begin{array}{l}\text { Highest Deliverable Capacity } \\
\qquad \mathrm{v}(\mathrm{STP}) / \mathrm{v}\end{array}$ & Number of Candidates \\
\hline acs & 183.89 & 817 \\
\hline cds & 189.64 & 512 \\
\hline dia & 173.28 & 75 \\
\hline hxg & 162.02 & 30 \\
\hline lvt & 175.16 & 720 \\
\hline nbo & 179.78 & 516 \\
\hline pcu & 191.07 & 61 \\
\hline rhr & 155.67 & 239 \\
\hline sod & 171.73 & 16 \\
\hline
\end{tabular}

The distribution of deliverable capacities of all molecules in nine nets is shown in Fig. 2. 
We find 48 MOF linkers, in four nets among the nine, having a higher deliverable capacity than MOF-5. We also show the structural formulas of linker molecules with highest methane deliverable capacity for each net in this figure. Our calculation of the deliverable capacity

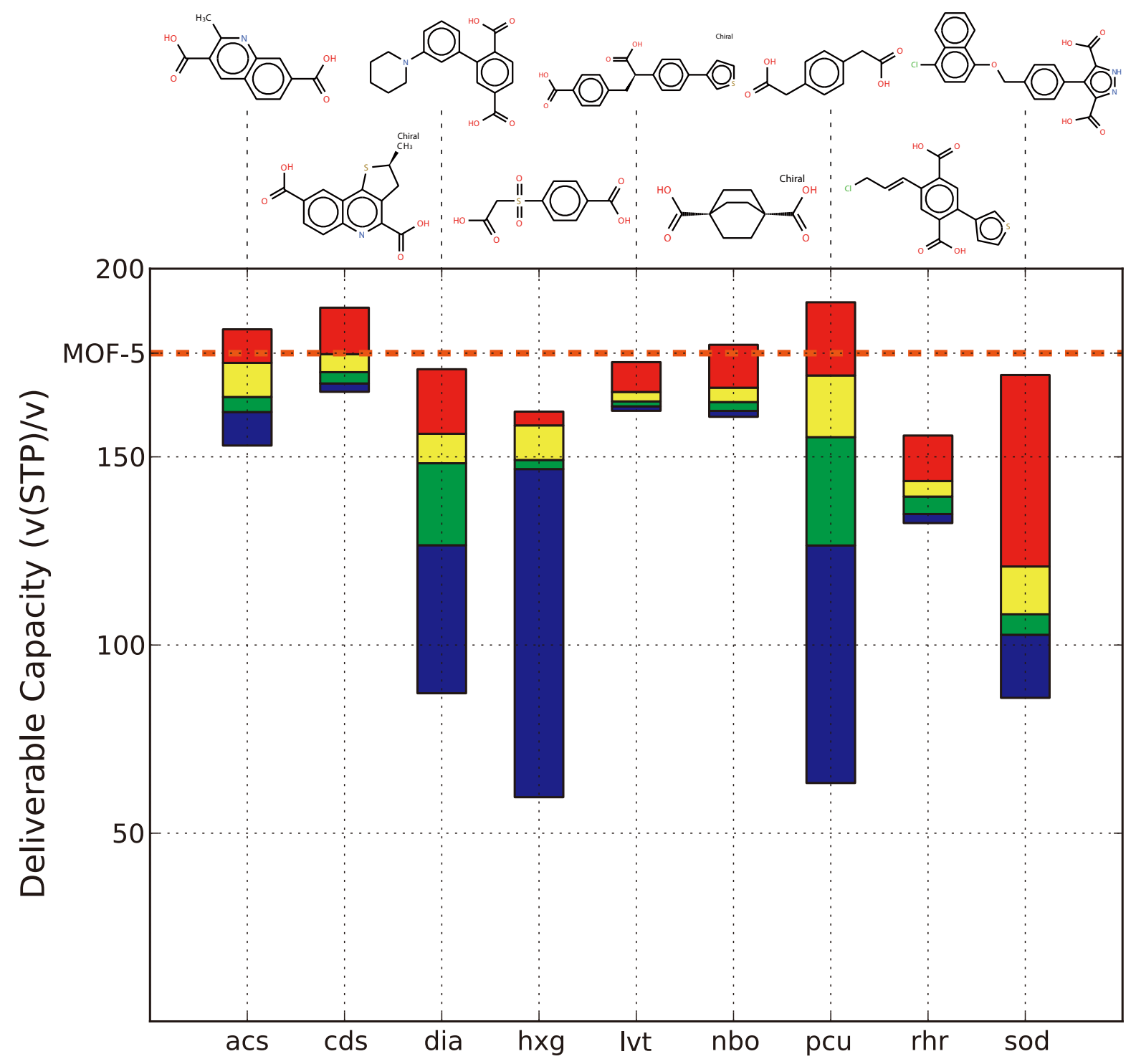

Figure 2: Bar plot of deliverable capacity of all molecules in final populations over nine nets. Our method finds 48 linkers with better deliverable capacity than MOF-5, in the nets of acs, cds, nbo, and pcu. The difficulty of constructing valid frameworks varies for different networks, and 100 linkers satisfying all constraints are not found for dia, hxg, pcu, and sod, as shown in Table. 1. The structural formulas of best linker molecules for each net is illustrated on the top of each bar. The different colors each represent one quartile of the data. 
of MOF-5 at these DOE target conditions is $177.6 \mathrm{v}(\mathrm{STP}) / \mathrm{v}$, nearly identical to the experimentally measured value of $177 \mathrm{v}(\mathrm{STP}) / \mathrm{v} \cdot{ }^{22}$ In pcu net alone we find eight linker molecules that outperform the $177.6 \mathrm{v}(\mathrm{STP}) / \mathrm{v}$ methane deliverable capacity of MOF-5, as shown in Fig. 3. Table 2 gives a few MOFs with the highest deliverable capacities in each of the nine



(a) pcu-191.07<smiles>O=C(O)CCCCC(=O)O</smiles>

(e) pcu-178.86

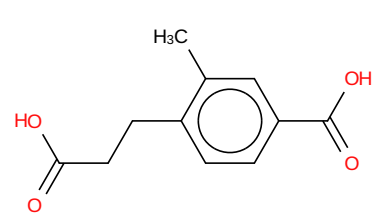

(b) pcu-183.74

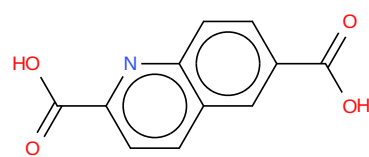

(f) pcu- 178.27

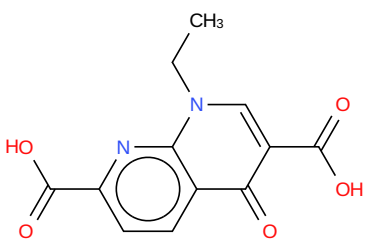

(c) pcu-180.53

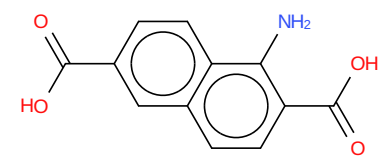

(g) pcu-177.78

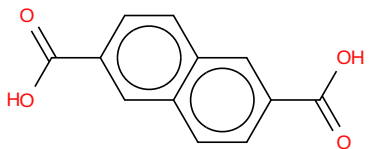

(d) pcu-179.98

(h) pcu-177.68

Figure 3: Representative structural formulas of the eight linker molecules in pcu net with higher deliverable capacity than MOF-5. Molecules are named as "net - deliverable capacity".

nets. Both SBUs and identified linker molecules are listed in this table.

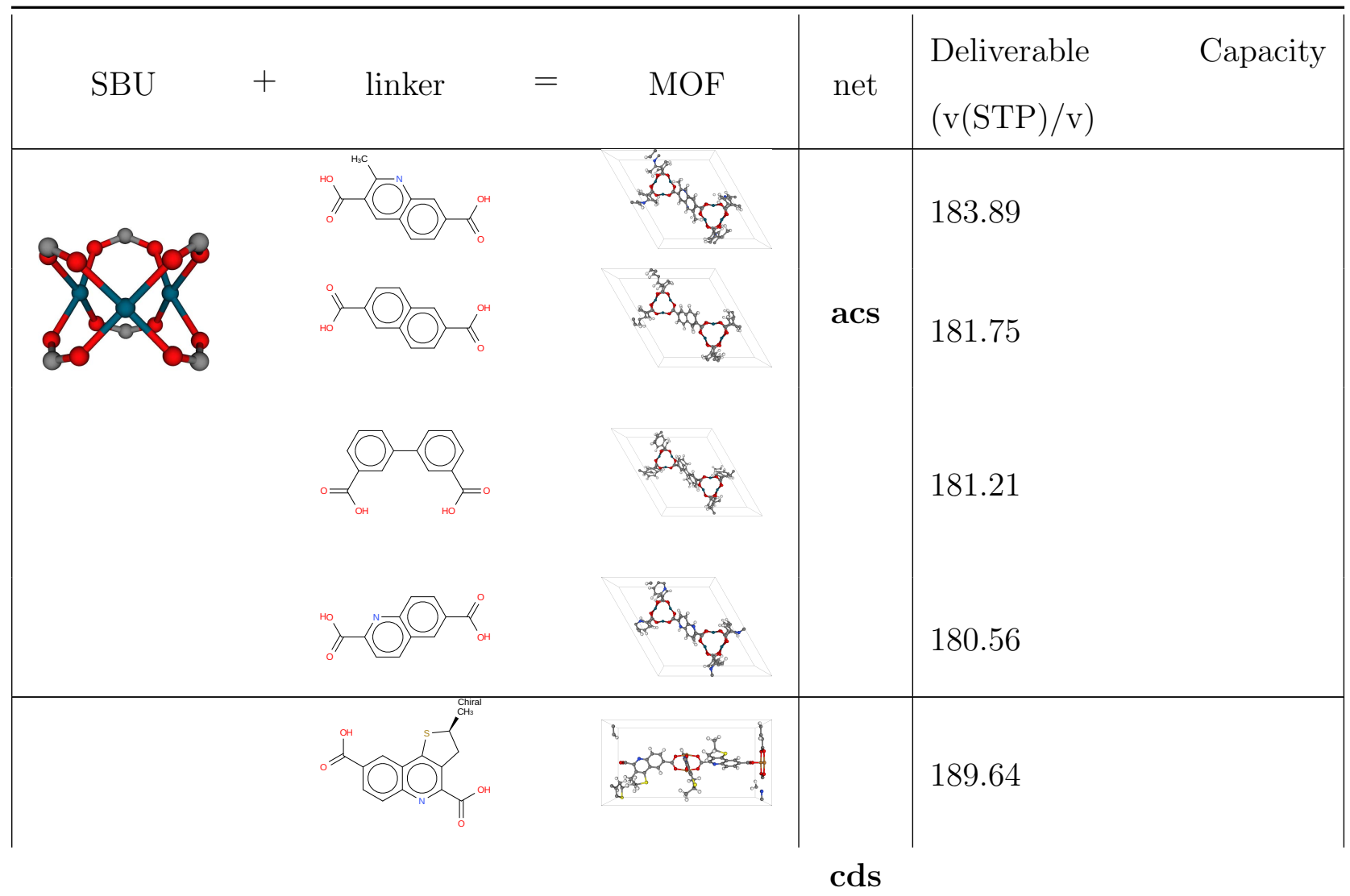




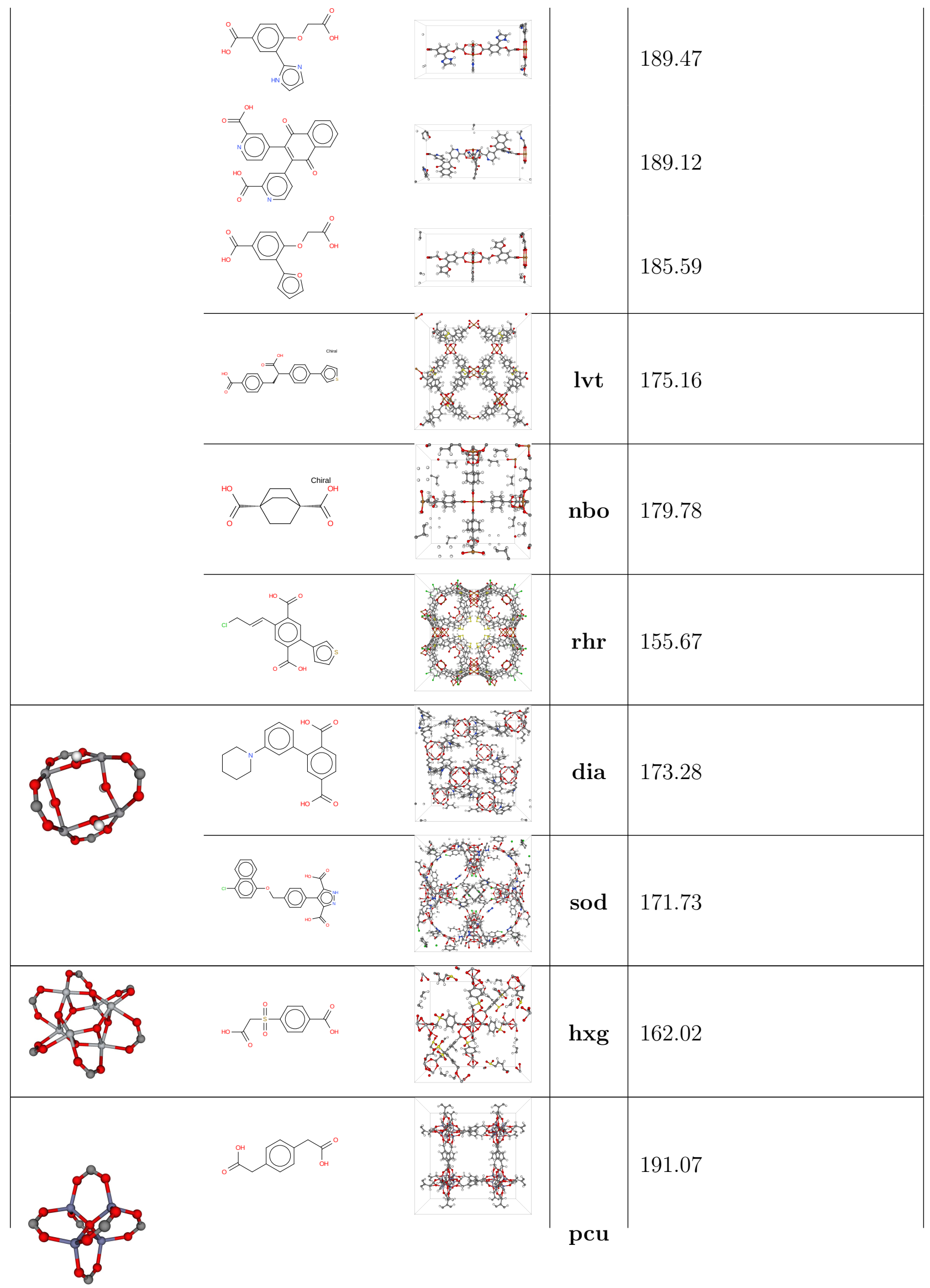





183.74
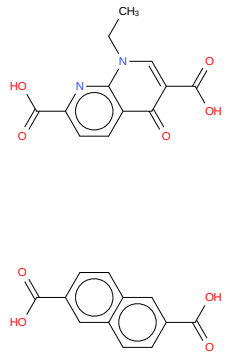

180.53

179.98

Table 2: A table of MOFs from predicted linker molecules and specified SBUs in the nine nets. Corresponding networks and calculated deliverable capacities are also listed. The SBUs are $\mathrm{Pd}_{3}$ for acs; $\mathrm{Cu}_{2}$ for cds, lvt, nbo and rhr; $\mathrm{V}_{4}(\mathrm{OH})_{4}$ for dia and sod; $\mathrm{Ti}_{6} \mathrm{O}_{6}$ for $\mathrm{hxg}$; and $\mathrm{Zn}_{4} \mathrm{O}$ for pcu. Each SBU figure with O-C-O connecting sites is depicted in a color scheme. Red: oxygen atoms; dark gray: carbon atoms; white: hydrogen atoms; other colors: respective metal atoms.

We record information of the synthetic route of each linker molecule in the population, along with what reactants are introduced from the precursor library and what chemical reactions are applied. One example of a synthetic route for a linker in the cds net is shown in Fig. 4.

We calculate the adsorption capacity of the 48 predicted MOFs in the four nets that outperform MOF-5. The one with highest deliverable capacity of each net is plotted in Fig. 5a. We characterize the diversity of the optimal MOFs by their modified Langmuir adsorption model parameters of Eq. (1). The fitted parameters of the MOFs are illustrated in Fig. 5b. The Henry's constants, $H=A_{\infty} / P_{1 / 2}$, which characterize the low-pressure adsorption, are shown in Fig. 5c. 


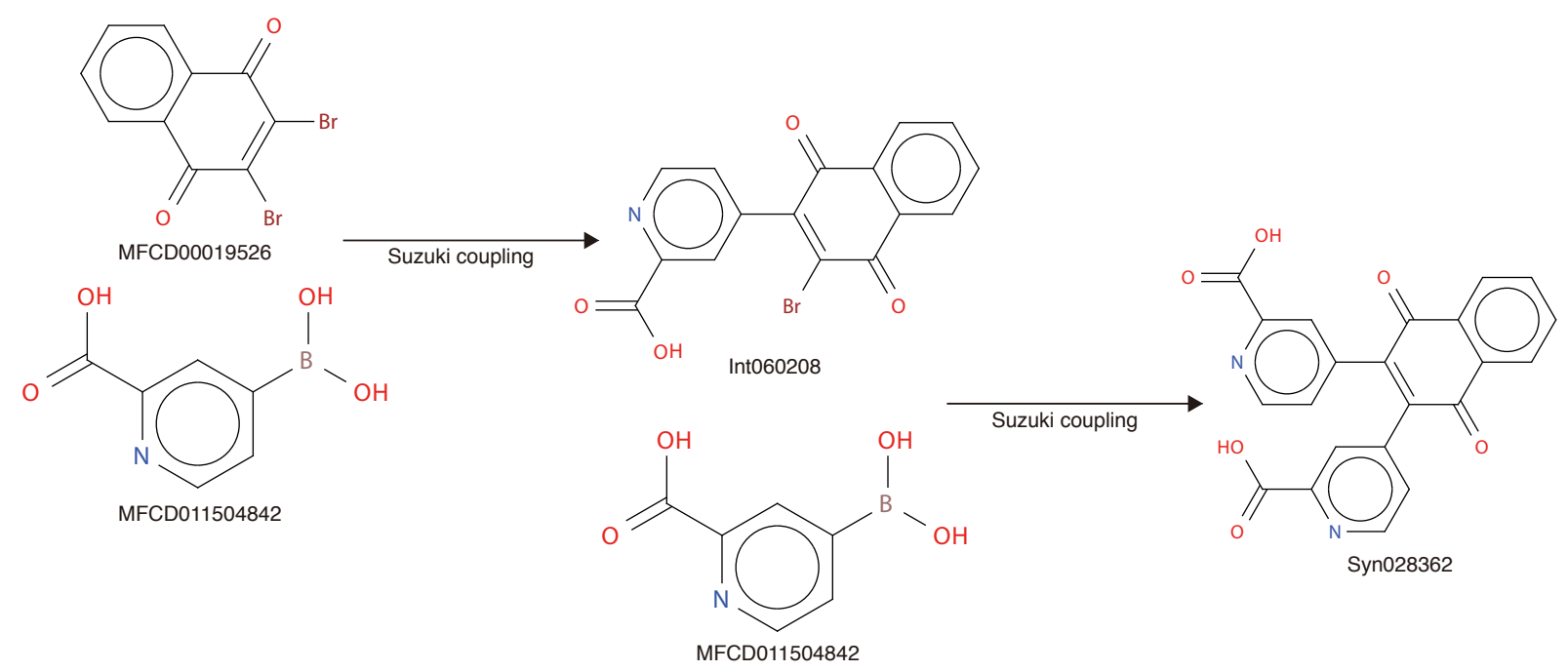

Figure 4: Synthetic route of a proposed linker Syn028362 in cds net. MFCD00019526 and MFCD011504842 are picked from the precursor library. They undergo a Suzuki coupling to produce the intermediate molecule Int060208. Int060208 undergoes another Suzuki coupling with precursor MFCD011504842 to produce the final product Syn028362.

\section{Discussion}

A guiding principle of our approach is to efficiently search the space of possible MOF compounds. We here focus on exploring the composition and conformation space of possible linkers. We find that to search this space, a sampling rather than enumeration approach proves more successful, echoing results founds in zeolite design. ${ }^{32}$ This efficient search of the MOF design space, analogous to the "biased Monte Carlo" methods that revolutionized computer simulation two decades ago, is a key methodological contribution of our work. We emphasize that we are not screening an existing database, but rather evolving MOFs in the composition space of all possibilities, subject to the known constraints of chemical synthesis and selecting for structures with predicted high deliverable capacity.

We found the known MOF-5 among the results, identified in the pcu net as pcu-177.55. MOF-5 and HKUST-1 are materials with long-standing records of the highest deliverable capacity, 177 and $182 \mathrm{v}(\mathrm{STP}) / \mathrm{v}$, respectively. ${ }^{22}$ UTSA-76a is an additional MOF with high deliverable capacity, but along with HKUST-1, is not accessible in any of the nine networks 


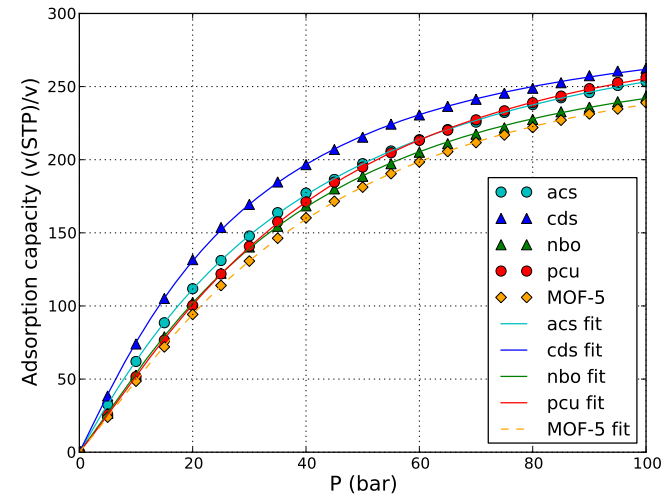

(a) Methane adsorption Isotherms

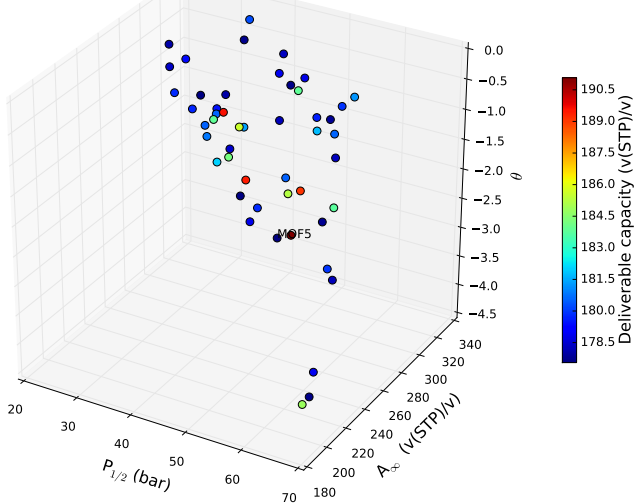

(b) Fitted parameters in the modified Langmuir isotherm model

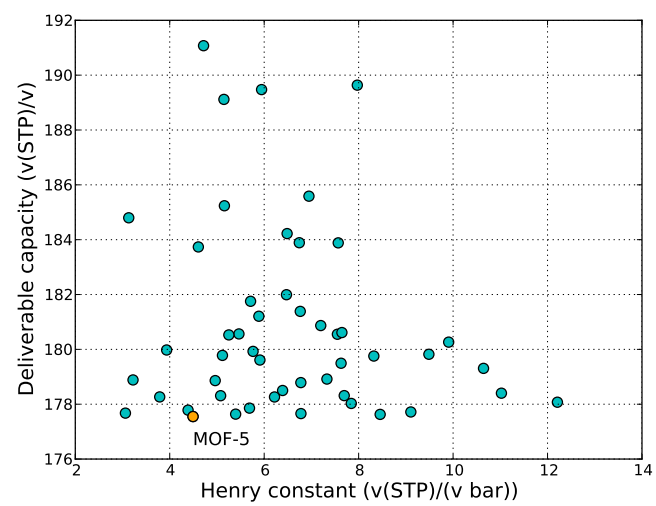

(c) Henry's constant

Figure 5: (a) Calculated methane adsorption isotherms of four predicted MOFs at $298 \mathrm{~K}$. MOF-5 is also shown as a reference (dashed). (b) The MOFs concentrate in a narrow band in the parameter space. (c) MOFs with the highest deliverable capacity $(>182 \mathrm{v}(\mathrm{STP}) / \mathrm{v})$ are found predominantly in the range of 3 to $8 \mathrm{v}(\mathrm{STP}) /(\mathrm{v}$ bar $)$ of Henry's constant. 
we consider. ${ }^{33}$ MOF-5 has been intensively studied and synthesized industrially via several approaches. ${ }^{20,34}$ We identified 48 MOFs with a predicted methane deliverable capacity greater than the well-studied MOF-5.

Recent studies in MOF design have identified structures with a variety of linker sizes, shapes, and chemistry. For instance, large porphyrins have been used as building blocks as MOF linkers. ${ }^{35}$ Long aromatic hydrocarbons, with up to 11 connected phenylene rings, have been used to construct IRMOF-74-XI. ${ }^{36}$ Numerous linkers for MTV-MOFs and MUF1-MUF-7 also contain long aromatic hydrocarbons. ${ }^{37,38}$ This diversity of linker chemistry suggests that de novo designed linkers using the approach introduced here by in silico exploration of chemical space may also be successfully incorporated into realistic MOFs. In this regard, the recent success of in silico design of an organic structure directing agent for the zeolite STW is also encouraging. ${ }^{39}$ Additional factors to be considered in experimental realizations of these predictions include solubility of a given linker in a relevant reaction solvent, variety of solvents, and temperature.

Structural properties of the linkers influence the likelihood of MOF self-assembly. Rigid linkers are often preferred to avoid an entropic penalty for incorporation of linkers into the MOF. Consequently, we require the standard deviation of pairwise distance, calculated over the set of molecular conformations, to be less than $0.5 \AA$. We also apply a filter on the number of torsions to eliminate linkers with excessive flexibility. As successful linkers are anticipated to be linear with anti-parallel attachment sites, we also apply the filter of mean pairwise angle. Loose filters tend to lead to more valid but mediocre candidates, while strict filters lead to fewer valid but outstanding candidates.

We notice that some evolved linker molecules have long side groups pendant to the backbone between the two site-connecting carboxylic groups. Intuitively, these side groups could help increase capacity because they provide more possible adsorption sites for gas molecules. The entropy of these side groups might inhibit MOF assembly if the configurational constraints on the side groups are significantly greater in the MOF than in solution. As long as 
these side groups are relatively unconstrained by SBUs, i.e. no collisions prevent framework construction, such side groups should not be notable barriers against MOF assembly. As the side groups become larger, they may prevent an efficient packing of methane. This situation is avoided by the optimization in the evolutionary linker search procedure. Additionally whether side groups actually increase deliverable capacity, and not simply adsorption at both 65 and 5.8 bar, is a subtle point that can only be answered by the detailed Monte Carlo calculations we performed.

The parameters of the modified Langmuir adsorption model were used to characterize the performance of predicted MOFs with adsorption capabilities exceeding MOF-5. Interestingly, as shown in Fig. 5b, we predict that MOFs optimal for deliverable capacity are in a limited region of the parameter space. Since for a given $P_{1 / 2}$ and $\theta$, larger deliverable capacities are produced by materials with larger $A_{\infty}$, the optimal materials shown in Fig. $5 \mathrm{~b}$ are those with the largest $A_{\infty}$ values at each $P_{1 / 2}$ and $\theta$. As shown in Fig. S1ab of Supplementary Information, there is a strong compensation effect: a decreasing $A_{\infty}$ or increasing $P_{1 / 2}$ is compenstated for by a more negative $\theta$.

Due to the stochastic nature of the in silico evolution, the algorithm does not necessarily form identical populations from a given net in each run. The deliverable capacity tends to increase with evolutionary time in a reproducible way in different runs (see Supplementary Information). Additionally, the highest deliverable capacity for each net does not fluctuate much between different runs. Finally, the in silico evolution for a given MOF network has a typical average computational cost. These observations suggest that the algorithm is computationally tractable, reliable, and can provide qualitatively consistent results.

\section{Conclusion}

We predicted MOFs that optimize methane gas deliverable capacity in nine networks, using a new in silico linker evolution procedure. Notably, 48 predicted linkers in the acs, cds, 
nbo, and pcu networks surpass MOF-5, a signature MOF with good methane deliverable capacity in a 65-5.8 bar pressure swing system at $298 \mathrm{~K}$. For each of the nine networks, we used a SBU known experimentally to provide the appropriate linker attachment sites. The predicted linkers were constrained to be rigid and to correctly form a MOF geometry. The predicted linkers have promising synthetic feasibility, as previous experience in drug

design with the in silico reactions considered here succeed in high yield $86 \%$ of the time. ${ }^{23}$ Clearly the predicted linkers are just one part of the puzzle, as solution conditions must still be identified for the linker and SBU to self-organize into the MOF. In this regard, we are encouraged by the recent success of this approach for novel zeolite synthesis. ${ }^{19}$

We have developed an evolutionary approach to explore the space of linker molecules in MOFs. This approach can be extended to design MOFs with multiple types of SBUs and multiple types of linkers. This approach is another step toward the creation of a materials genome of MOFs with designed properties. Our work is the first to use an in silico evolution procedure to create chemically synthesizable linkers that are evolved to optimize MOF methane deliverable capacity.

\section{Acknowledgments}

This research was supported by the US Department of Energy, Office of Basic Energy Sciences, Division of Chemical Sciences, Geosciences and Biosciences under Award DE-FG0212ER16362.

\section{References}

(1) Yaghi, O. M.; Li, G.; Li, H. Selective Binding and Removal of Guests in a Microporous MetalOrganic Framework. Nature 1995, 378, 703-706.

(2) Furukawa, H.; Cordova, K. E.; OKeeffe, M.; Yaghi, O. M. The Chemistry and Applications of Metal-Organic Frameworks. Science 2013, 341, 974-986. 
(3) Meek, S. T.; Greathouse, J. A.; Allendorf, M. D. Metal-Organic Frameworks: A Rapidly Growing Class of Versatile Nanoporous Materials. Advanced Materials 2011, 23, 249267.

(4) Horcajada, P.; Gref, R.; Baati, T.; Allan, P. K.; Maurin, G.; Couvreur, P.; Férey, G.; Morris, R. E.; Serre, C. Metal-Organic Frameworks in Biomedicine. Chem. Rev. 2012, 112, 1232-1268.

(5) Sarkisov, L. Toward Rational Design of Metal-Organic Frameworks for Sensing Applications: Efficient Calculation of Adsorption Characteristics in Zero Loading Regime. J. Chem. Phys. C 2012, 116, 3025-3033.

(6) Kreno, L. E.; Leong, K.; Farha, O. K.; Allendorf, M.; Duyne, R. P. V.; Hupp, J. T. Metal-Organic Framework Materials as Chemical Sensors. Chem. Rev. 2012, 112, 11051125.

(7) Li, J.-R.; Sculley, J.; Zhou, H.-C. Metal-Organic Frameworks for Separations. Chem. Rev. 2012, 112, 869-932.

(8) Herm, Z. R.; Wiers, B. M.; Mason, J. A.; van Baten, J. M.; Hudson, M. R.; Zajdel, P.; Brown, C. M.; Masciocchi, N.; Krishna, R.; Long, J. R. Separation of Hexane Isomers in a Metal-Organic Framework with Triangular Channels. Science 2013, 340, 960-964.

(9) Cychosz, K. A.; Ahmadab, R.; Matzger, A. J. Liquid Phase Separations by Crystalline Microporous Polymers. Chem. Sci. 2010, 1, 293-302.

(10) Lee, J.-Y.; Farha, O. K.; Roberts, J.; Scheidt, K. A.; Nguyen, S.-B. T.; Hupp, J. T. Metal-Organic Framework Materials as Catalysts. Chem. Soc. Rev. 2009, 38, 14501459.

(11) Suh, M. P.; Park, H. J.; Prasad, T. K.; Lim, D.-W. Hydrogen Storage in Metal-Organic Frameworks. Chem. Rev. 2012, 112, 782-835. 
(12) Getman, R. B.; Bae, Y.-S.; Wilmer, C. E.; Snur, R. Q. Review and Analysis of Molecular Simulations of Methane, Hydrogen, and Acetylene Storage in Metal-Organic Frameworks. Chem. Rev. 2012, 112, 703-723.

(13) Li, J.-R.; Ma, Y.; McCarthy, M. C.; Sculley, J.; Yu, J.; Jeong, H.-K.; Balbuena, P. B.; Zhou, H.-C. Carbon Dioxide Capture-Related Gas Adsorption and Separation in MetalOrganic Frameworks. Coordination Chemistry Reviews 2011, 255, 1791-1823.

(14) Wilmer, C. E.; Leaf, M.; Lee, C. Y.; Farha, O. K.; Hauser, B. G.; Hupp, J. T.; Snurr, R. Q. Large-Scale Screening of Hypothetical MetalOrganic Frameworks. Nature Chemistry 2012, 4, 83-89.

(15) Draznieks, C. M.; Newsam, J. M.; Gorman, A. M.; Freeman, C. M.; Férey, G. De Novo Prediction of Inorganic Structures Developed through Automated Assembly of Secondary Building Units (AASBU Method). Angew. Chem. Int. Ed. 2000, 39, 22702275 .

(16) Farha, O. K.; Yazaydn, A. Ö.; Eryazici, I.; Malliakas, C. D.; Hauser, B. G.; Kanatzidis, M. G.; Nguyen, S. T.; Snurr, R. Q.; Hupp, J. T. De novo Synthesis of a MetalOrganic Framework Material Featuring Ultrahigh Surface Area and Gas Storage Capacities. Nature Chemistry 2010, 2, 944-948.

(17) Smit, B.; Frenkel, D. Understanding Molecular Simulation: From Algorithms to Applicatons, 2nd ed.; Academic Press: San Diego, 2012.

(18) Kutchukian, P. S.; Shakhnovich, E. I. De Novo Design: Balancing Novelty and Confined Chemical Space. Expert Opin. Drug Discov. 2010, 5, 789-812.

(19) Pophale, R.; Daeyaert, F.; Deem, M. W. Computational Prediction of Chemically Synthesizable Organic Structure Directing Agents for Zeolites. J. Mater. Chem. A 2013, 1, 6750-6760. 
(20) Li, H.; Eddaoudi, M.; O'Keeffe, M.; Yaghi, O. M. Design and Synthesis of an Exceptionally Stable and Highly Porous Metal-Organic Framework. Nature 1999, 402, 276-279.

(21) Eddaoudi, M.; Kim, J.; Rosi, N.; Vodak, D.; Wachter, J.; O’Keeffe, M.; Yaghi, O. M. Systematic Design of Pore Size and Functionality in Isoreticular MOFs and Their Application in Methane Storage. Science 2002, 295, 469-472.

(22) Mason, J. A.; Veenstra, M.; Long, J. R. Evaluating MetalOrganic Frameworks for Natural Gas Storage. Chemical Science 2014, 5, 32-51.

(23) Vinkers, H. M.; de Jonge, M. R.; Daeyaert, F. F. D.; Heeres, J.; Koymans, L. M. H.; van Lenthe, J. H.; Lewi, P. J.; Timmerman, H.; Aken, K. V.; Janssen, P. A. J. SYNOPSIS: SYNthesize and OPtimize System in Silico. J. Med. Chem. 2003, 46(13), 2765-2733.

(24) Liu, J.; Lukose, B.; Shekhah, O.; Arslan, H. K.; Weidler, P.; Gliemann, H.; Bräse, S.; Grosjean, S.; Godt, A.; Feng, X.; et al. A Novel Series of Isoreticular Metal Organic Frameworks: Realizing Metastable Structures by Liquid Phase Epitaxy. Sci. Rep. 2012, 2, 921.

(25) Willems, T. F.; Rycroft, C. H.; Kazi, M.; Meza, J. C.; Haranczyk, M. Algorithms and Tools for High-Throughput Geometry-Based Analysis of Crystalline Porous Materials. Micropor. Mesopor. Mater. 2012, 149, 134-141.

(26) Martin, R. L.; Haranczyk, M. Construction and Characterization of Structure Models of Crystalline Porous Polymers. Cryst. Growth Des. 2014, 14(5), 2431-2440.

(27) Rappé, A. K.; Casewit, C. J.; Colwell, K. S.; Goddard, W. A., III; Skiff, W. M. UFF, a Full Periodic Table Force Field for Molecular Mechanics and Molecular Dynamics Simulations. J. Am. Chem. Soc. 1992, 114, 10024-10035. 
(28) Daeyaert, F.; De Jonge, M.; Koymans, L.; Vinkers, M. An Ant Algorithm for the Conformational Analysis of Flexible Molecules. Journal of Computational Chemistry 2007, 28, 890-898.

(29) Ponder, J. W.; Richards, F. M. An Efficient Newton-Like Method for Molecular Mechanics Energy Minimization of Large Molecules. Journal of Computational Chemistry 1987, 8, 1016-1024.

(30) Simon, C. M.; Kim, J.; Lin, L.-C.; Martin, R. L.; Haranczyk, M.; Smit, B. Optimizing Nanoporous Materials for Gas Storage. Phys. Chem. Chem. Phys. 2014, 16, 5499-5513.

(31) Flower, D. R. On the Properties of Bit String-Based Measures of Chemical Similarity. J. Chem. Inf. Comput. Sci. 1998, 38, 379-386.

(32) Pophale, R.; Daeyaert, F.; Deem, M. W. A Database of New Zeolite-Like Materials. Phys. Chem. Chem. Phys 2011, 13, 12407-12412.

(33) Li, B.; Wen, H.-M.; Wang, H.; Wu, H.; Tyagi, M.; Yildirim, T.; Zhou, W.; Chen, B. A Porous MetalOrganic Framework with Dynamic Pyrimidine Groups Exhibiting Record High Methane Storage Working Capacity. J. Am. Chem. So. 2014, 136, 6207-6210.

(34) Mueller, U.; Schubert, M.; Teich, F.; Puetter, H.; Schierle-Arndta, K.; Pastré, J. MetalOrganic Frameworks-Prospective Industrial Applications. Journal of Materials Chemistry 2006, 16, 626-636.

(35) Kandambeth, S.; Shinde, D. B.; Panda, M. K.; Lukose, B.; Heine, T.; Banerjee, R. Enhancement of Chemical Stability and Crystallinity in Porphyrin-Containing Covalent Organic Frameworks by Intramolecular Hydrogen Bonds. Angew. Chem. Int. Ed. 2013, 52, 13052-13056.

(36) Furukawa, H.; Cordova, K. E.; O'Keeffe, M.; Yaghi, O. M. The Chemistry and Applications of Metal-Organic Frameworks. Science 2013, 341(6149), 1230444. 
(37) Deng, H.; Doonan, C. J.; Furukawa, H.; Ferreira, R. B.; Towne, J.; Knobler, C. B.; Wang, B.; Yaghi, O. M. Multiple Functional Groups of Varying Ratios in Metal-Organic Frameworks. Science 2010, 327, 846-850.

(38) Liu, L.; Konstas, K.; Hill, M. R.; Telfer, S. G. Programmed Pore Architectures in Modular Quaternary Metal-Organic Frameworks. J. Am. Chem. Soc. 2013, 135, 1773117734 .

(39) Schmidt, J. E.; Deem, M. W.; Davis, M. E. Synthesis of a Specified, Silica Molecular Sieve using Computationally Predicted Organic Structure Directing Agents. Angew. Chem. Int. Ed. 2014, 53, 8372-8374. 


\section{Graphical TOC Entry}

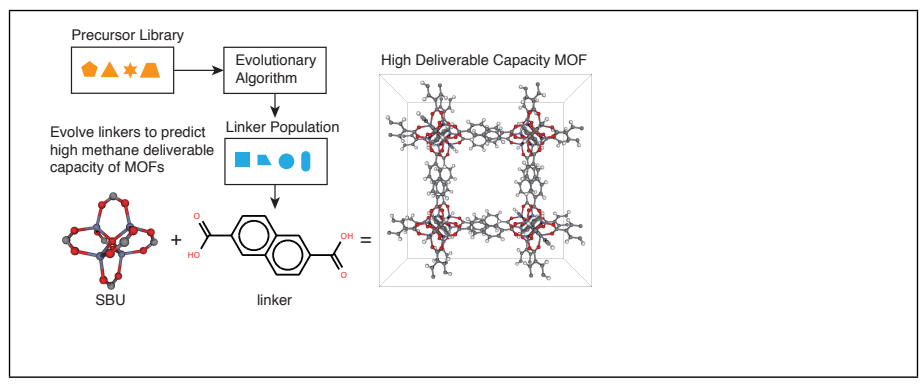

